\title{
RELAÇÃO DE ESCOLARIDADE, FAIXA ETÁRIA E PROFISSÃO DE MÃES COM A OFERTA DE CHUPETA E MAMADEIRA A SEUS FILHOS
}

\section{Relationship of schooling level, age group and mother profession with offer of pacifier and baby's bottles to your children}

\author{
Kelly Cristina Alves Silvério ${ }^{(1)}$, Ana Paula Silva Ferreira (2), Cinthia Mara Johanns (2), \\ Aline Wolf ${ }^{(3)}$, Ana Maria Furkim ${ }^{(4)}$, Jair Mendes Marques ${ }^{(5)}$
}

\begin{abstract}
RESUMO
Objetivo: avaliar a relação do grau de escolaridade, faixa etária e a profissão das mães com a oferta de chupeta e mamadeira a seus filhos. Método: participaram deste estudo 190 mães de crianças entre 0 e 10 anos de idade, com idades de 20 a 45 anos que responderam a um questionário com questões fechadas a respeito de profissão, nível de escolaridade e uso de chupeta e mamadeira de seus filhos. Resultados: não foi observada diferença significante entre o grau de escolaridade quanto ao uso de chupeta $(p=0,915)$. O uso de mamadeira foi significantemente maior nos filhos de mães com ensino superior $(p=0,0124)$. Não houve diferença significante quanto à faixa etária das mães e a presença desses hábitos nos filhos (chupeta: $p=0,427$; mamadeira: $p=0,427$ ); e, entre mães que trabalham em casa e que trabalham fora de casa (chupeta: $p=0,061$; mamadeira: $p=0,798$ ). Não houve diferença entre mães que trabalham na área da saúde e em outras áreas (chupeta: $p=0,339$; mamadeira: $p=0,455)$. Conclusão: as mães com nível superior de escolaridade oferecem significantemente mais mamadeira aos seus filhos, o que não ocorreu quanto ao uso de chupeta. Não foi observada diferença significante quanto ao uso de chupeta e mamadeira em relação à faixa etária e profissão de mães.
\end{abstract}

DESCRITORES: Hábitos; Escolaridade; Idade Materna, Mães

(1) Fonoaudióloga; Docente do Departamento de Fonoaudiologia da Faculdade de Odontologia de Bauru da Universidade de São Paulo, Bauru - FOB/USP, Bauru, SP, Brasil; Doutora em Ciências pela Faculdade de Odontologia de Piracicaba da Universidade Estadual de Campinas - FOP/ UNICAMP.

(2) Fonoaudióloga; Mestre em Distúrbios da Comunicação pela Universidade Tuiuti do Paraná, UTP, Curitiba, PR, Brasil.

(3) Fonoaudióloga; Docente do Departamento de Oftalmologia e Otorrinolaringologia, Curso de Fonoaudiologia da Faculdade de Medicina da Universidade de São Paulo, Ribeirão Preto - FMRP/USP, Ribeirão Preto, SP, Brasil; Doutora em Ciências Biomédicas pela Universidade Estadual de Campinas - UNICAMP.

(4) Fonoaudióloga; Professora Adjunto do Curso de Fonoaudiologia da Universidade Federal de Santa Catarina, Florianópolis, PR, Brasil; Doutora em Distúrbios da Comunicação Humana pela Universidade Federal de São Paulo UNIFESP.

(5) Matemático; Docente do Programa de Mestrado e Doutorado em Distúrbios da Comunicação da Universidade Tuiuti do Paraná,UTP, Curitiba, PR, Brasil; Doutor em Ciências Geodésicas pela Universidade Federal do Paraná.

Conflito de interesses: inexistente

\section{INTRODUÇÃO}

Os hábitos são padrões de contração muscular aprendidos, de natureza complexa, que se diferenciam dos hábitos orais anormais e deletérios por interferirem no padrão regular do crescimento facial ${ }^{1}$. Tal crescimento ocorre de acordo com as características genéticas de cada indivíduo, tendo como fatores determinantes a hereditariedade, desnutrição, doenças e clima².

Os hábitos orais deletérios são comumente definidos como padrões neuromusculares atípicos que podem determinar, entre outras coisas, o desenvolvimento das más oclusões, devido aos prejuízos que ocasionam, principalmente, na posição dos dentes, desencadeando alterações, também, no sistema estomatognático, pois introduzem forças estranhas a esse. As pesquisas realizadas nesta área relatam teorias que buscam explicar a etiologia dos hábitos 
deletérios referentes à sucção não nutritiva, hábitos de morder e hábitos funcionais. Esses podem ser de origem fisiológica, emocional ou aprendida ${ }^{3} \mathrm{e}$ seus prejuízos serão determinados, em especial, pela freqüência, intensidade, duração e característica do objeto utilizado, bem como, a idade do sujeito na época de instalação dos hábitos ${ }^{4-8}$.

Geralmente, só são notados no ambiente familiar quando trazem algum dano estético e/ou de fala ${ }^{9}$. Têm início na primeira infância por meio de hábitos anormais como: sucção não nutritiva (sucção digital, sucção de chupeta e de mamadeira), morder - objetos, sucção e mordida do lábio, onicofagia, bruxismo; hábitos funcionais deglutição atípica, interposição lingual, respiração oral, má postura durante o sono, bruxismo diurno e noturno, deslocamento mandibular lateral por contatos prematuros ${ }^{10,11}$.

Alguns estudos ${ }^{12-15}$ pesquisaram sobre a utilização da chupeta e relataram que a mesma é utilizada pelos pais para acalmar a criança principalmente na hora do choro. Além disso, os autores constataram que a maioria das mães já possui a chupeta antes mesmo de ir à maternidade, pois consideram os hábitos de chupeta e mamadeira normais, fazendo parte da rotina da criança ${ }^{8,16}$, talvez pela falta de conhecimento sobre as possíveis alterações que podem ser ocasionadas por esses hábitos ${ }^{17,18}$. Outro fator relevante, em relação ao uso da chupeta, é a maior incidência deste hábito em crianças cujas mães têm baixa escolaridade ${ }^{1,16}$.

Em pesquisa realizada com mães que apresentaram hábitos orais deletérios ${ }^{14}$ os autores constataram que a mãe que possui o mesmo hábito que o filho, torna mais difícil a eliminação do mesmo, ficando mais condescendente.

Diversos estudos têm sido realizados em relação aos hábitos orais deletérios da infância e suas possíveis conseqüências no equilíbrio do sistema estomatognático, ${ }^{5,19-25}$ porém, pouco descrevem sobre o perfil das mães de crianças que possuem estes hábitos.

Frente ao exposto, este estudo teve como objetivo avaliar a relação do grau de escolaridade, faixa etária e a profissão de mães com a oferta de chupeta e mamadeira a seus filhos.

\section{MÉTODO}

Participaram desta pesquisa, 190 mães de crianças com idades entre zero e 10 anos, advindas de cidades do Estado do Paraná, com idades de 18 a 45 anos (média = 32,5 anos; Desvio Padrão $=5,5$ ). Foram incluídas mães cujas crianças tinham desenvolvimento neuropsicomotor normal, ausência de alterações genéticas, síndromes ou malformações e que não tinham passado por intervenção cirúrgica na região craniofacial. Nenhum critério em relação à idade das mães foi estabelecido.

Todas as mães selecionadas receberam um questionário que continha questões fechadas sobre: 1. grau de escolaridade - ensino fundamental, médio, superior ou pós-graduação; 2 . idade; 3. profissão: a mãe deveria declarar a profissão, caso trabalhasse fora de casa ou declarar "do lar", caso não trabalhasse fora de casa; 4 . declarar se ofereceu chupeta e mamadeira ao seu filho. As mães que declararam ter mais de um filho, responderam ao questionário apenas uma vez, escolhendo sobre qual filho estaria relatando as informações. Os pesquisadores não controlaram essa variável.

Este estudo foi previamente aprovado pelo Comitê de Ética em Pesquisa da Universidade Tuiuti do Paraná, sob número de protocolo 00067/2008. Depois de esclarecidos todos os detaIhes da pesquisa, todos os participantes assinaram o Termo de Consentimento Livre e Esclarecido.

Após a coleta, os dados foram organizados para tratamento estatístico e comparação entre as variáveis, separando as mães por: a) escolaridade: ensino fundamental e médio (65 mães), ensino superior e pós-graduação (125 mães); b) faixa etária: 18 a 30 anos (66 mães), 31 a 40 anos (103 mães) e mais de 40 anos (21 mães); c) profissão: área da saúde - médica, fonoaudióloga, fisioterapeuta, enfermeira, nutricionista, dentista (25 mães) -, outras áreas - engenheira, arquiteta, secretária, advogada, vendedora (145 mães) - e "do lar" (20 mães). Na área da saúde, compararam-se também as mães "fonoaudiólogas" (13 mães) com as mães das demais áreas da saúde (12 mães).

A Figura a seguir revela o número de mães, segundo as categorias de análise propostas:

\begin{tabular}{|lc|}
\hline Categorias & $\begin{array}{c}\text { Número } \\
\text { de mães }\end{array}$ \\
\hline Escolaridade & \\
Ensino fundamental e médio & 65 \\
Ensino superior e pós-graduação & 125 \\
\hline Faixa etária & \\
18 a 30 anos & 66 \\
31 a 40 anos & 103 \\
Mais de 40 anos & 21 \\
\hline Profissão & \\
Área da saúde & 25 \\
Fonoaudiólogas & 13 \\
Outras áreas & 145 \\
Do lar & 20 \\
\hline
\end{tabular}

Figura1 - Distribuição de mães de acordo com as categorias de análise 
Os dados foram tratados estatisticamente, comparando-se a escolaridade das mães com o uso de chupeta e mamadeira; faixa etária das mães com o uso de chupeta e mamadeira de seus filhos; profissão das mães com o uso de chupeta e mamadeira. Para estas análises foi aplicado o Teste estatístico de diferenças de proporções, com nível de significância de 0,05.

\section{RESULTADOS} 1 a 4.

Os resultados encontram-se nas tabelas de

Tabela 1 - Comparação do nível de escolaridade das mães de acordo com uso de chupeta e mamadeira dos filhos

\begin{tabular}{lccc}
\hline & \multicolumn{3}{c}{ Escolaridade da Mãe } \\
\cline { 2 - 4 } Hábitos do filho & $\begin{array}{c}\text { Ensino Fundamental } \\
\text { e/ou Médio }\end{array}$ & $\begin{array}{c}\text { Ensino Superior ou } \\
\text { Pós-graduação }\end{array}$ & Valor de $\mathbf{p}$ \\
& $\mathbf{N}(\mathbf{\%})$ & $\mathbf{N}(\mathbf{\%})$ & 0,9153 \\
\hline Uso de Chupeta & $39(60,0)$ & $74(59,2)$ & $0,0124^{\star}$ \\
Uso de Mamadeira & $54(83,0)$ & $118(94,4)$ & \\
\hline
\end{tabular}

Análise: Teste diferença de proporções $\left(p \geq 0,05^{\star}\right)$

Tabela 2 - Comparação da faixa etária das mães de acordo com o uso de chupeta e mamadeira dos filhos

\begin{tabular}{|c|c|c|c|c|c|c|}
\hline \multirow{3}{*}{$\begin{array}{l}\text { Hábito } \\
\text { do filho }\end{array}$} & \multicolumn{3}{|c|}{ Faixa etária da mãe } & \multicolumn{3}{|c|}{ Valor de $p$} \\
\hline & $\begin{array}{c}18 \text { a } 30 \text { anos } \\
\text { (I) }\end{array}$ & $\begin{array}{l}31 \text { a } 40 \text { anos } \\
\text { (II) }\end{array}$ & $\begin{array}{c}\text { Mais de } \\
40 \text { anos (III) }\end{array}$ & \multirow[t]{2}{*}{ (I) e (II) } & \multirow[t]{2}{*}{ (I) e (III) } & \multirow[t]{2}{*}{ (II) e (III) } \\
\hline & $\mathrm{N} \quad(\%)$ & N (\%) & N (\%) & & & \\
\hline $\begin{array}{l}\text { Uso de } \\
\text { chupeta }\end{array}$ & $40(60,6)$ & $55(53,4)$ & $16(76,2)$ & 0,3587 & 0,1971 & 0,0566 \\
\hline $\begin{array}{l}\text { Uso de } \\
\text { mamadeira }\end{array}$ & $59(89,4)$ & $94(91,3)$ & $19(90,5)$ & 0,6808 & 0,7953 & 0,9065 \\
\hline
\end{tabular}

Análise: Teste diferença de proporções $\left(p \geq 0,05^{\star}\right)$

A tabela 3 evidencia os dados da comparação da profissão das mães de acordo com o uso de chupeta e mamadeira de seus filhos. Não houve diferença significante entre as mães profissionais da área da saúde e mães denominadas "do lar", mães da área da saúde e de outras áreas e quando comparadas mães "do lar" e de outras áreas que não a saúde.

A tabela 4 traz a comparação da profissão de mães de outras áreas da saúde e Fonoaudiologia, de acordo com o uso de chupeta e mamadeira dos filhos e evidencia que não houve diferença significante entre os dados.
A tabela 1 traz os resultados da comparação do nível de escolaridade das mães de acordo com uso de chupeta e mamadeira de seus filhos. Foi possível observar que mães de nível de escolaridade superior e pós-graduação oferecem significantemente mais mamadeira a seus filhos do que mães com nível de escolaridade fundamental e médio.

A tabela 2 compara a faixa etária das mães de acordo com o uso de chupeta e mamadeira de seus filhos e não evidenciou diferença significante quando comparadas as diferentes faixas etárias: 20 a 30 anos e 31 a 40 anos, 20 a 30 e mais de 40 anos, 31 a 40 e mais de 40 anos. 
Tabela 3 - Comparação da profissão das mães de acordo com o uso de chupeta e mamadeira dos filhos

\begin{tabular}{|c|c|c|c|c|c|c|}
\hline \multirow[b]{2}{*}{$\begin{array}{l}\text { Hábito } \\
\text { do filho }\end{array}$} & \multicolumn{3}{|c|}{ Profissão da mãe } & \multicolumn{3}{|c|}{ Valor de $p$} \\
\hline & $\begin{array}{l}\text { Área de } \\
\text { saúde (I) } \\
\begin{array}{cc}\mathrm{N} & (\%)\end{array}\end{array}$ & $\begin{array}{c}\text { “Do lar” (II) } \\
N \quad(\%)\end{array}$ & \begin{tabular}{cc}
\multicolumn{2}{c}{ Outras (III) } \\
$\mathbf{N} \quad(\%)$
\end{tabular} & (I) e (II) & (I) e (III) & (II) e (III) \\
\hline $\begin{array}{l}\text { Uso de } \\
\text { chupeta }\end{array}$ & $13(52,0)$ & $8(40,0)$ & $90(62,1)$ & 0,4271 & 0,3399 & 0,0616 \\
\hline $\begin{array}{l}\text { Uso de } \\
\text { mamadeira }\end{array}$ & $24(96,0)$ & $18(90,0)$ & $133(91,7)$ & 0,4271 & 0,4559 & 0,7982 \\
\hline
\end{tabular}

Análise: Teste diferença de proporções $\left(p \geq 0,05^{\star}\right)$

Tabela 4 - Comparação da profissão de mães - outras da área da saúde e Fonoaudiologia - de acordo com o uso de chupeta e mamadeira dos filhos

\begin{tabular}{lccc}
\hline \multirow{2}{*}{ Hábitos do filho } & \multicolumn{3}{c}{ Profissão da área da saúde } \\
\cline { 2 - 4 } & $\begin{array}{r}\text { Outras da saúde } \\
\mathbf{N}(\mathbf{\%})\end{array}$ & $\begin{array}{c}\text { Fonoaudiologia } \\
\mathbf{N}\end{array}$ & \multirow{2}{*}{ Valor de $\mathbf{p}$} \\
\hline Uso de chupeta & $6(50,00)$ & $7(53,84)$ & 0,8432 \\
Uso de mamadeira & $3(25,00)$ & $8(61,53)$ & 0,0790 \\
\hline
\end{tabular}

Análise: Teste diferença de proporções $\left(p \geq 0,05^{\star}\right)$

pais com o objetivo de confortar a criança, principalmente na hora do choro, porém não há registros sobre a origem e a multiplicação deste hábito no meio social. Alguns autores ${ }^{12,13}$ ao pesquisarem sobre as possíveis causas e conseqüências da utilização da chupeta relataram que a freqüência de uso de chupetas em crianças brasileiras é bastante elevada e que a maioria das mães já possui a chupeta antes mesmo de ir à maternidade. Desta forma, o nível de escolaridade das mães parece não influenciar no uso de chupeta de seus filhos uma vez que as mães parecem assumir o uso da chupeta como algo natural e necessário para confortar a criança.

Em relação ao uso de mamadeira, foi possível observar que foi significantemente maior entre as mães com ensino superior ou pós-graduação ( $p=$ 0,0124 ) - tabela 1 , sendo que $94,4 \%$ das mães com nível de escolaridade superior ou com pós-graduação relataram oferecer mamadeira aos seus filhos, enquanto que $83,1 \%$ das mães com nível de escolaridade menor relataram o mesmo fato. Constata-se, portanto, que o nível de escolaridade não é fonte de conhecimento de fatores prejudiciais do uso da mamadeira, nem tão pouco pode ser considerado responsável pela não instalação desse hábito, pois seria esperado que quem apresentasse maior escolaridade supostamente saberia mais sobre os prejuízos que causa o uso da mamadeira. Outros fatores podem influenciar esta escolha: comodidade, praticidade e menor tempo para alimentar a criança.

Em uma pesquisa sobre a família e os hábitos orais na infância ${ }^{7}$ os autores relataram que a maioria das mães pesquisadas considera os hábitos de chupeta e mamadeira normais. O uso da mamadeira por um período prolongado, como visto em outro estudo ${ }^{16}$, pode ser explicado pela falta de conhecimento dos pais sobre os malefícios que ela traz, como por exemplo, na oclusão dentária ${ }^{3,5-10,13,19,21}$, alimentação ${ }^{15-17} \mathrm{e}$ até mesmo na fala ${ }^{23}$. Além disso, o fato de cada vez mais, a mulher estar envolvida com o trabalho, o tempo disponível com os filhos torna-se menor. Alguns autores ${ }^{25}$ relataram que os hábitos orais podem ser influenciados, assim como outros comportamentos, por alguns fatores sociais, como emprego da mãe que colabora na renda familiar de forma parcial ou total.

Quanto à faixa etária, não houve diferença significante entre as mães e os hábitos de chupeta e mamadeira de seus filhos quando comparadas as diferentes faixas etárias - Tabela 2. Entretanto, observa-se uma tendência à significância $(p=0,0566)$ das mães com mais de 40 anos oferecerem chupeta a seus filhos quando comparadas 
às mães da faixa etária de 31 a 40 anos. Segundo estudo ${ }^{17}$ que objetivou observar os cuidados populares adotados por mães adolescentes na assistência aos filhos nos seis primeiros meses de vida, as mães adolescentes apesar de receberem orientações de profissionais da saúde com relação à mamadeira, acabam a utilizando devido a influência de suas mães, ao contrário do uso da chupeta onde as adolescentes relataram que nem sempre levam em conta os conselhos e as experiências de suas mães.

Em relação à profissão, não houve diferença significante entre as mães profissionais da área da saúde, outras áreas e mães "do lar" e os hábitos de chupeta e mamadeira de seus filhos Tabela 3. Entretanto, observou-se uma tendência à significância $(p=0,0616)$ quando comparadas mães de outras profissões com mães "do lar", ou seja, mais mães de outras profissões parecem oferecer chupeta a seus filhos do que mães consideradas "do lar". Seria esperado que mães que trabalham em casa apresentassem menor incidência de relatos quanto ao uso de chupeta e mamadeira de seus filhos, por terem maior disponibilidade de tempo com as crianças e com possibilidade de freqüentar programas de aleitamento materno que estimulam a diminuição do uso de chupeta e mamadeira, prevenindo possíveis alterações no sistema estomatognático, o que não ocorreu no presente estudo. Porém, autores ${ }^{1}$ relataram que algumas variáveis como a renda familiar, escolaridade materna, trabal ho materno e ocupação dos pais podem estar relacionados a condições de saúde infantil. Os autores ressaltaram a necessidade de se obter dados estatísticos para caracterizar o perfil das crianças e dos pais cujos filhos utilizaram chupeta e mamadeira.

Interessante observar também que quando comparadas mães da área da saúde e fonoaudiólogas que seriam supostamente consideradas os profissionais mais críticos em relação à oferta de chupeta e mamadeira para seus filhos, não foi observada diferença significante para ambos ( $p=0,8432$ e $p=0,0790$, respectivamente). Embora esta diferença não tenha ocorrido de forma significante, surpreendentemente, $61,53 \%$ das mães fonoaudiólogas oferecem mamadeira a seus filhos, enquanto que apenas $25 \%$ das mães ligadas a demais áreas da saúde o fazem, observando-se uma tendência à significância $(p=0,0790)$, ou seja, mais mães fonoaudiólogas oferecem mamadeira a seus filhos quando comparadas às mães de outras profissões da área da saúde. Talvez o baixo número de sujeitos da amostra relacionada às profissões da área da saúde e fonoaudiologia não tenha tornado possível observar diferença significante.

\section{CONCLUSÃO}

Os dados do presente estudo permitiram concluir que mães com nível superior de escolaridade oferecem significantemente mais mamadeira aos seus filhos, o que não ocorreu quanto ao uso de chupeta. Não foi observada diferença significante quanto ao uso de chupeta e mamadeira em relação à faixa etária e profissão de mães.

\section{ABSTRACT}

Purpose: to evaluate the relationships among schooling level, age group and mother profession with offer of pacifier and baby's bottles to your children. Method: 190 mothers with ages varying from 20 to 45 having children from 0 to 10 took part in this study. Mothers answered a questionnaire with questions regarding profession, schooling level and use of nipples and baby's bottles by their children. Results: no significant differences were found between mothers having primary or secondary education and mothers with higher education regarding the use of nipples $(p=0.915)$. On the other hand, baby's bottle use was significantly higher among children of mothers with higher education $(p=0.0124)$. No significant difference regarding age group of mothers and the presence of these habits in children or between mothers who work in the house and those who work outside as regards the use of nipples $(p=0.427 ; p=0.061)$ and baby's bottles $(p=0.427 ; p=0.798)$. We also observed no difference among mothers who work in the health area and those working in other fields (nipples: $p=0.339$; baby's bottles: $p=0.455)$. Conclusion: mothers with higher education offer significantly more baby's bottles to their children, but not nipples. No significant difference was observed as for the use of nipples and baby's bottles as related to age group and mothers' profession.

KEYWORDS: Habits; Education Status; Maternal Age; Mothers 


\section{REFERÊNCIAS}

1. Fracasso MLC, Marchi V, Goya S, Provenzano MGA, Takahashi K. Perfil das Mães e Crianças Frequentadoras do Programa Clínica de Bebês no núcleo Integrado de Saúde NIS III Iguaçu em Maringá - Pr. Revista Saúde e Pesquisa. 2008; 1(3): 325-9.

2. Katz CR, Rosenblatt A, Gondim PP. Nonnutritive sucking habits in Brazilian children: effects on deciduous dentition and relationshipwith facial morphology. Am J Orthod Dentofacial Orthop. 2004; 126(1): 53-7.

3. Junqueira P. Amamentação, Hábitos Orais E Mastigação: Orientações, Cuidados e Dicas. 3ª . ed. Rio de Janeiro: Revinter; 2005. 33 p.

4. Gonçalves LPV, Toledo OA, Otero SAM. Relação entre bruxismo, fatores oclusais e hábitos bucais. Dental Press J. Orthod. 2010; 15(2): 97-104.

5. Fofano CSN, Mialhe FL, Silva RP, Brum SC. Conhecimentos, atitudes e práticas maternas em relação ao uso da chupeta. Pesq Bras Odontoped Clin Integr. 2009; 9(1):119-23.

6. Gimenez CMM, Moraes ABA, Bertoz AP, Bertoz FA, Ambrosano GB. Prevalência de más oclusões na primeira infância e sua relação com as formas de aleitamento e hábitos infantis. Rev. Dent. Press Ortodon. Ortopedi. Facial. 2008; 13(2):70-83.

7. Almeida RVD, Nogueira Filho JJ, Jardim MCAM. Prevalência de Maloclusão e sua relação com hábitos bucais deletérios em escolares. Rev Pesq Bras Odontoped Clin Integr. 2002; 2(1):43-5.

8. Heimer MV, Tornisiello Katz CR, Rosenblatt A. Non-nutritive sucking habits, dental malocclusions, and facial morphology in Brazilian children: a longitudinal study. 2008; Eur J Orthod. 30(6):580-5. 9. Caglar E, Larsson E, Andersson EM, Hauge MS, Ogaard B, Bishara S, et al. Feeding, artificial sucking habits, and malocclusions in 3-year-old girls in different regions of the world. J Dent Child. 2005; 72(1): 25-30.

10. Ovsenik M. Incorrect orofacial functions until 5 years of age and their association with posterior crossbite. Am J Orthod Dentofacial Orthop. 2009; 136(3):375-81.

http://dx.doi.org/10.1590/S1516-18462011005000117

RECEBIDO EM: 22/12/2010

ACEITO EM: 21/04/2011

Endereço para correspondência:

Kelly C. A. Silverio

Al. Dr. Octávio Pinheiro Brisolla, 9-75

Bauru - SP

CEP: 17012-101

E-mail: kcsilver@terra.com.br

kellysilverio@usp.br
11. Adair SM. Pacifier use in children: a review of recent literature. Pediatr Dent. 2003;25(5):449-58

12. Victora CG, Behague DP, Barros FC, Olinto MT, Weiderpass E. Pacifier use and short breastfeeding duration: cause, consequence, or coincidence? Pediatrics. 1997; 99:445-53.

13. Tomita NE, Bijella VT, Franco LJ. Relação entre hábitos bucais e má-oclusão em pré-escolares. Rev Saúde Pública. 2000; 3(34): 299-303.

14. Serra-Negra JMC, Vilela LC, Rosa AR, Andrade ELSP, Paiva SM, et al. Hábitos Bucais Deletérios: os filhos imitam as mães na adoção destes hábitos? Revista Odonto Ciência - Fac. Odonto/PUCRS. 2006; 21(52): 146-52.

15. Araújo CMT, Silva GAP, Coutinho SB. Aleitamento materno e uso da chupeta: repercussões na alimentação e no desenvolvimento do sistema sensório motor oral. Rev Paul Pediatria. 2007; 25(1): 59-65.

16. Soares, MEM, Giugliani ERJ, Braun ML, Salgado ACN, Oliveira AP, Aguiar PR. Uso de chupeta e sua relação com o desmame precoce em população de crianças nascidas em Hospital Amigo da Criança. Jornal de Pediatria. 2003; 79(4):309-16.

17. Trawitzki LVV, Lima ATW, Melchior MO, Grechi $\mathrm{TH}$, Valera FCP. Aleitamento e hábitos orais deletérios em respiradores orais e nasais. Rev Bras Otorrinol. 2005; 71(6): 747-51.

18. Tomeleri KR, Marcon SS. Práticas populares de mães adolescentes no cuidado aos filhos. Acta Paul Enferm. 2009; 22(3)272-80.

19. Peres KG, Barros AJD, Peres MA, Cictora CG. Effects of breastfeeding and sucking habits on malocclusion in a birth cohort study. Revista de Saúde Publica. 2007; 41(3): 343-50.

20. Degan VV, Puppin-Rontani RM. Prevalence of pacifier-sucking habits and successful methods to eliminate them: a preliminary study. J Dent Child. 2004; 71(2):148-51.

21. Kulkarni GV, Lau D. A single appliance for the correction of digit-sucking, tongue-thrust, and posterior cross bite. Pediatr Dent. 2010; 32(1):61-3. 22. Castelo PM, Gavião MB, Pereira LJ, Bonjardim LR. Relationship between oral parafunctional/ nutritive sucking habits and temporomandibular joint dysfunction in primary dentition. Int $\mathrm{J}$ Paediatr Dent. 2005; 15(1):29-36.

23. Barbosa C, Vasquez S, Parada MA, Gonzalez JCV, Jackson C, Yanez ND, et al. The relationship of bottle feeding and other sucking behavior with speech disorder in Patagonian preschoolers. BMC Pediatr. 2009; 9: 66.

24. Medeiros AP, Ferreira JT, Felicio CM. Correlation between feeding methods, non-nutritive sucking and orofacial behavior. Pró-Fono. 2009; 21(4): 315-9.

25. Cavassani VGS, Ribeiro SG, Nemr NK, Greco AM, Köhle J, Lehn CN. Hábitos orais de sucção: estudo piloto em população de baixa renda. Rev Bras Otorrinolaringol. 2003; 69:(1)106-10. 